\title{
Wavelength dependence of photon- induced photoacoustic streaming technique for root canal irrigation
}

Nejc Lukač

Jure Zadravec

Peter Gregorčič

Matjaž Lukač

Matija Jezeršek 


\title{
Wavelength dependence of photon-induced photoacoustic streaming technique for root canal irrigation
}

\author{
Nejc Lukač, ${ }^{\mathrm{a}, \star}$ Jure Zadravec, ${ }^{a}$ Peter Gregorčič, ${ }^{a}$ Matjaž Lukač, ${ }^{b}$ and Matija Jezeršek ${ }^{\mathrm{a}}$ \\ aUniversity of Ljubljana, Faculty of Mechanical Engineering, Askerceva 6, 1000 Ljubljana, Slovenia \\ ${ }^{\mathrm{b} I n s t i t u t e}$ Josef Stefan, Jamova 39, 1000 Ljubljana, Slovenia
}

\begin{abstract}
Laser-enhanced irrigation of complex root canals appears to be a very promising technique to improve the outcome of root canal treatment. This applies, in particular, if the technique can be effective at very low laser energies in irrigating not only the main canal but also the small lateral canals. This is important in order to avoid potential undesirable effects at higher laser energies such as temperature increase, dentin ablation, or extrusion of irrigating solution beyond the apical foramen. An improved understanding of the role of laser parameters, such as laser wavelength and pulse duration, in irrigation of lateral canals is therefore desired in order to optimize treatment efficacy. The influence of laser wavelength and pulse duration on cavitation phenomena was studied using shadow photography and a method of measuring fluid flow in lateral canals based on tracking of movements of small air bubbles naturally forming in liquid as a result of laser agitation. A simulated model of a root canal including a narrow lateral canal designed to represent typical root canal morphology was used for the water flow measurements. The following three laser wavelengths with relatively high absorption in water were studied: Er:YAG $(2.94 \mu \mathrm{m}), \mathrm{Er}, \mathrm{Cr}: Y S G G(2.73 \mu \mathrm{m})$, and Nd:YAP $(1.34 \mu \mathrm{m})$. Among the three wavelengths studied, the Er:YAG laser wavelength was found to be the most effective in formation of cavitation bubbles and in generating fluid motions within narrow lateral canals. A comparison between the shadow photography and fluid motion data indicates that it is the bubble's radius and not the bubble's volume that predominantly influences the fluid motion within lateral canals. Based on the results of our study, it appears that effective minimally invasive laser-assisted irrigation can be performed with low Er:YAG laser pulse energies below $10 \mathrm{~mJ}$. ๑ 2016 Society of Photo-Optical Instrumentation Engineers (SPIE) [DOI: 10.1117/1.JBO.21.7.075007]
\end{abstract}

Keywords: endodontics; Er,Cr:YSGG; Er:YAG; irrigant fluid motion; Nd:YAP.

Paper 160235R received Apr. 12, 2016; accepted for publication Jun. 16, 2016; published online Jul. 11, 2016.

\section{Introduction}

Root canal treatment may be a very challenging procedure due to the highly variable and complex root canal anatomy. ${ }^{1}$ Accessory canals, apical deltas, isthmi, irregular-shaped canals, and root canal curvatures are some of the anatomic aberrations that influence the diagnosis and treatment effectiveness. ${ }^{2}$

Root canal infections are caused by different species of bacteria, fungi, and viruses that invade the root canal system. ${ }^{3,4} \mathrm{~A}$ typical root canal treatment consists of using mechanical files and/or rotary instruments to enlarge and clean the root canal, followed by applying different chemical irrigants to remove the resulting smear layer ${ }^{5-11}$ and disinfect the root canal system. The most commonly used chemical irrigants are $\mathrm{NaOCl}$ and EDTA solutions. ${ }^{12-16}$ However, their application has been found to be effective in the middle and cervical thirds of the root canal wall, but not in the apical part. ${ }^{17}$ Even more importantly, conventional syringe irrigation has been found not to effectively debride and disinfect isthmi, cul-de-sacs, lateral canals, and apical ramifications, regardless of the chemical used. ${ }^{2}$ It is also difficult to rinse any remaining debris out of the anatomic irregularities before filling the root canal with an inert material. ${ }^{13}$

Different agitation techniques have been introduced with the goal of improving the efficacy of irrigation solutions, including agitation with gutta-percha cones, hand files, plastic instruments, and ultrasonic devices. ${ }^{10}$ More recently, the use of laser energy has been proposed to enhance irrigation by the transfer of pulsed energy into the irrigation liquid. ${ }^{18-21}$

Laser-activated agitation is typically based on a photoninduced photoacoustic streaming (PIPS) procedure using short bursts of Er:YAG laser energy with a wavelength of $\sim 3 \mu \mathrm{m} .{ }^{21-24}$ In this procedure, laser pulses are delivered into the fluid-filled pulp chamber using a fiber tip (FT). Due to its very high absorption coefficient in $\mathrm{OH}$ containing liquids, ${ }^{25}$ the Er:YAG pulse light is absorbed within a liquid layer only $\sim 1 \mu \mathrm{m}$ thick. Consequently, the liquid is locally and instantly heated over its boiling point and a vapor bubble starts to develop at the FT's end. ${ }^{26-29}$ The vapor bubble first expands and then collapses after reaching its maximum volume $\left(V_{\max }\right)$. Under certain conditions, the collapse initiates a secondary bubble's growth. This turbulent photoacoustic agitation of irrigants moves the fluid three-dimensionally throughout the root canal system, actively pumps the tissue debris out of the canals, and is expected to clean and disinfect not only the main but also lateral canals. ${ }^{30,31}$

When performing laser-activated agitation, it is advantageous to use the lowest energy pulses where the technique is still effective. This is to avoid dentin ablation, extrusion of irrigating solution beyond the apical foramen, ${ }^{32}$ and to minimize temperature increase of the irrigation fluid. In this paper, we report on an 
experimental study of the dependence of the optodynamic (OD) laser energy-conversion efficiency ${ }^{28}$ on the activating laser wavelength and how this dependence may affect laser irrigation of root canals.

In the first part of the study, we measured the dependence of the bubble size and energy on laser wavelength in an infinite liquid reservoir. The OD energy-conversion efficiency of three laser wavelengths was studied: Er:YAG $(2.94 \mu \mathrm{m})$, Er, Cr:YSGG $(2.73 \mu \mathrm{m})$, and Nd:YAP $(1.34 \mu \mathrm{m})$. Measurements in the infinite liquid were performed in order to be able to distinguish the influence of the laser wavelength from other parameters that may influence bubble formation dynamics in a complex root canal anatomy. In a liquid reservoir formed by a root canal, the dynamics of the cavitation bubble is more complex and depends on the length, diameter, and shape of the main root canal and its side canals. ${ }^{33}$ The measured bubble formation in the infinite liquid thus represents an ideal case where the OD energy-conversion efficiency is affected only by the laser parameters such as the laser wavelength while in a real root canal the liquid reservoir boundaries only further reduce this energyconversion efficiency.

In the second part of the study, we applied an experimental technique to measure the influence of the activating laser wavelength (Er:YAG and Nd:YAP) on the speed of the fluid flow in a simulated model of a root canal including a narrow lateral canal.

\section{Materials and Methods}

The methods used to record the effects of PIPS were high-speed shadow photography ${ }^{28,34}$ and video acquisition of liquid motion utilizing a high speed camera. The main advantages of photography are the better quality and higher resolution of obtained images as well as much better time resolution achieved by short illumination times. The main disadvantage of photography is the relatively slow frequency of image acquisition, rendering its use impractical in documenting single, transitory events, such as the flow of liquid through a lateral canal caused by PIPS. For this reason, a high-speed, high-resolution camera was used to capture the motion of liquid through a simulated narrow lateral canal. A two-way ANOVA was used to determine the statistical significance of the results (analysis performed with STATA).

\subsection{Shadow Photography}

The experimental setup for shadow photography is schematically shown in Fig. 1, and it is basically the same as described in Refs. 28, 35.

The cavitation bubbles generated in liquid by an excitation laser were illuminated by $30 \mathrm{ps}$ long frequency-doubled $\mathrm{Nd}$ : YAG $(\lambda=532 \mathrm{~nm})$ laser pulses (Ekspla, Lithuania, PL2250SH-TH), and imaged through a microscope by a charge-coupled device (CCD) camera (Basler AG, Germany, scA1400-17fm, $1.4 \mathrm{Mpx}$ ). The spatial resolution of the optical system was in the range of 3.6 to $5.6 \mu \mathrm{m} /$ pixel. For a particular series of measurements, the spatial resolution was determined using images of a calibrating pattern. The excitation and illumination lasers as well as the camera were synchronized with a signal generator (SG; Tektronix, US, AFG 3102) connected to a personal computer (PC).

Three types of excitation laser sources were used: Er:YAG $(2.94 \mu \mathrm{m})$, Er,Cr:YSGG $(2.73 \mu \mathrm{m})$, and Nd:YAP $(1.34 \mu \mathrm{m})$. The Er:YAG system used was a LightWalker AT (manufactured by Fotona), the Er,Cr:YSGG laser was a WaterLase iPlus (manufactured by Biolase), and the Nd:YAP laser was a modified Spectro XP (manufactured by Fotona). The erbium lasers were fitted with appropriate standard FT handpieces (Fotona Er: YAG H14 handpiece with $400 \mu \mathrm{m}$ PIPS tip; Biolase Er,Cr: YSGG Gold handpiece with $415 \mu \mathrm{m}$ RFT3 tip), while the

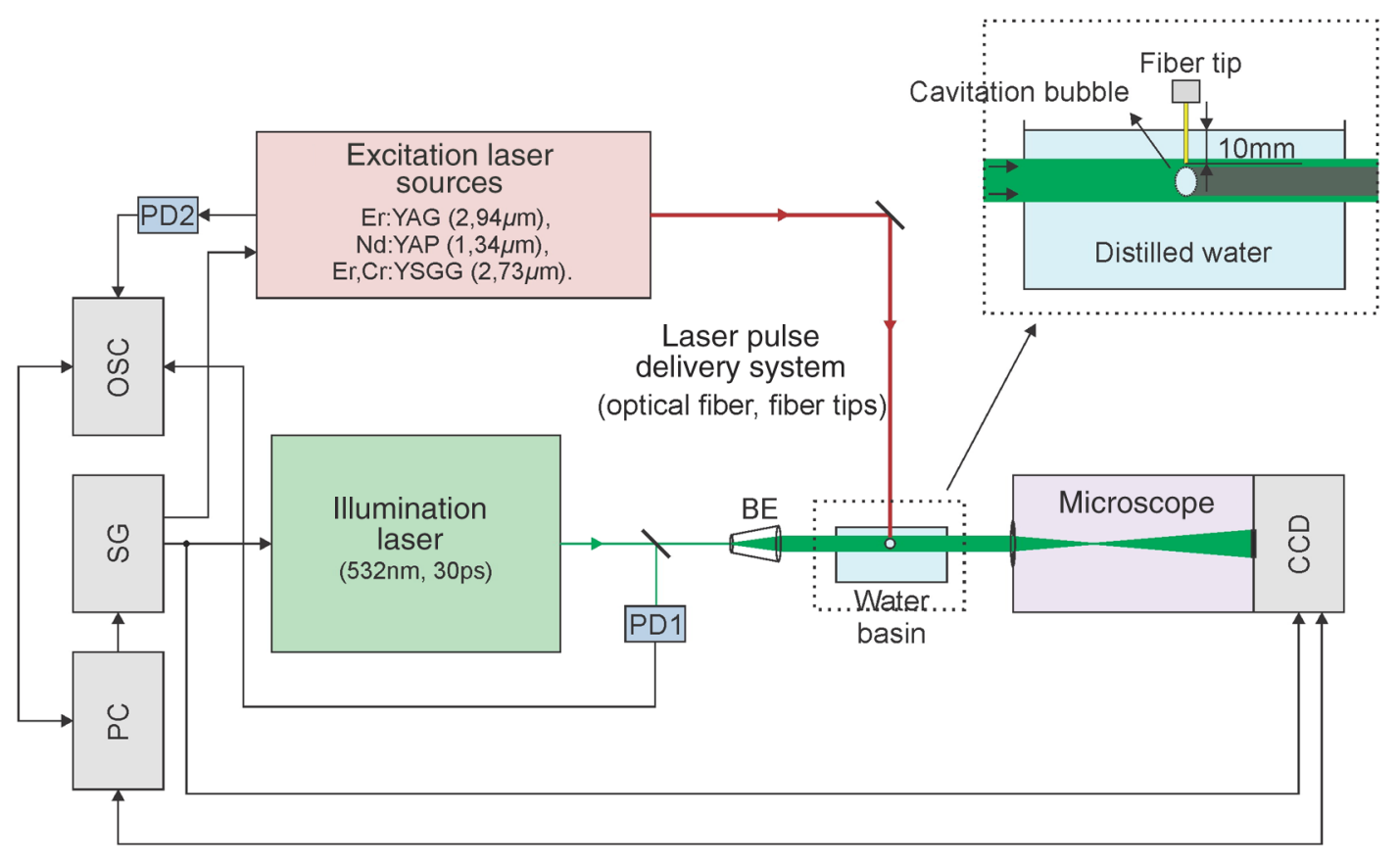

Fig. 1 Schematic overview of the shadow photography experimental setup. A SG is used to trigger the CCD camera, the excitation lasers and the illumination laser. A beam expander (BE) is used to increase the diameter of the illumination beam. Two photodiodes (PD1 and PD2) and an oscilloscope (OSC) are used to detect the output of the illumination and the excitation laser, respectively. The process is controlled via a PC. 
Nd:YAP laser was fitted with a laboratory handpiece ending with the PIPS $400 \mu \mathrm{m}$ tip. The PIPS and RFT3 tips have a conical ending resulting in a radial emission out of the FT.

The lasers were operated in the following pulse modes and durations: (a) Er:YAG laser in standard SSP $(80 \mu \mathrm{s})$ mode, ${ }^{36}$ (b) Er,Cr:YSGG laser in $\mathrm{H}$ pulse $(400 \mu \mathrm{s}),{ }^{36}$ and $\mathrm{S}$ pulse $(2000 \mu \mathrm{s})$ duration mode, and (c) Nd:YAP laser in $90-\mu \mathrm{s}$ pulse duration mode. The above pulse durations $\left(t_{\mathrm{L}}\right)$ represent times during which $90 \%$ of the cumulative laser pulse energy was delivered to the liquid, as measured with a $60-\mathrm{MHz}$ InAs photodiode (PD-InAs) at laser pulse energy $E_{\mathrm{L}} \approx 100 \mathrm{~mJ}$.

The excitation laser pulses were delivered into a large reservoir $(100 \times 100 \times 70 \mathrm{~mm})$ filled with distilled water through a corresponding FT. The FT's end was placed $h=10 \mathrm{~mm}$ below the water level, as shown schematically in the inset of Fig. 1.

Using shadow photography, we measured the vapor-bubble dynamics for different laser wavelengths, pulse duration modes, and pulse energies, $E_{\mathrm{L}}$. A sequence of images from multiple events was recorded by varying the time delay between the excitation pulse and the illumination pulse. The bubble locally changes the refractive index of the liquid. Consequently, a discontinuity appears on its wall, representing the boundary between the steam and the surrounding water. This results in the refraction of the laser beam casting a shadowgraph where the FT and cavitation bubble are visible as a black area on a bright background. ${ }^{34}$
A typical sequence of images of a bubble captured at different times after the beginning of a laser pulse is shown in Fig. 2. The laser-energy deposition causes water to superheat, and its explosive boiling induces a vapor bubble. As already observed elsewhere, ${ }^{28}$ a spherical bubble develops when a conical FT is used while a channel-like bubble develops when a flat FT is used. All FTs used in this shadowgraphic experiment (PIPS and RFT3) had a conical ending, and, therefore, spherical bubbles were generated. The spherical bubble's volume $\left(V=4 \pi R^{3} / 3\right.$ ) was calculated from the bubble's radius $(R)$ as obtained using the image processing of the shadowgraphs captured by the CCD camera.

After the laser-induced boiling, the high pressure of the vapor leads to the rapid expansion of the bubble's radius $(R)$, observed in images from 0 to $100 \mu$ s in Fig. 2. During the expansion, the bubble passes over the equilibrium state. Thus, at its maximum radius $\left(R_{\max }\right)$ and maximal volume $\left(V_{\max }=4 \pi R_{\max }^{3} / 3\right)$ (see Fig. 2 at $120 \mu \mathrm{s}$ ), the internal pressure is lower than the pressure in the surrounding liquid. This difference in pressures forces the bubble to collapse (e.g., see images from 140 to $240 \mu \mathrm{s}$ in Fig. 2). A shockwave is emitted at the time of the bubble's collapse (see Fig. 2 at $260 \mu$ s). The collapse, in turn, can initiate secondary oscillations of the bubble, as visible from 280 to $440 \mu$ s in Fig. 2.

When the bubble reaches its maximum volume, its radial velocity vanishes. The total energy of the bubble $E_{\mathrm{B}}$ can thus

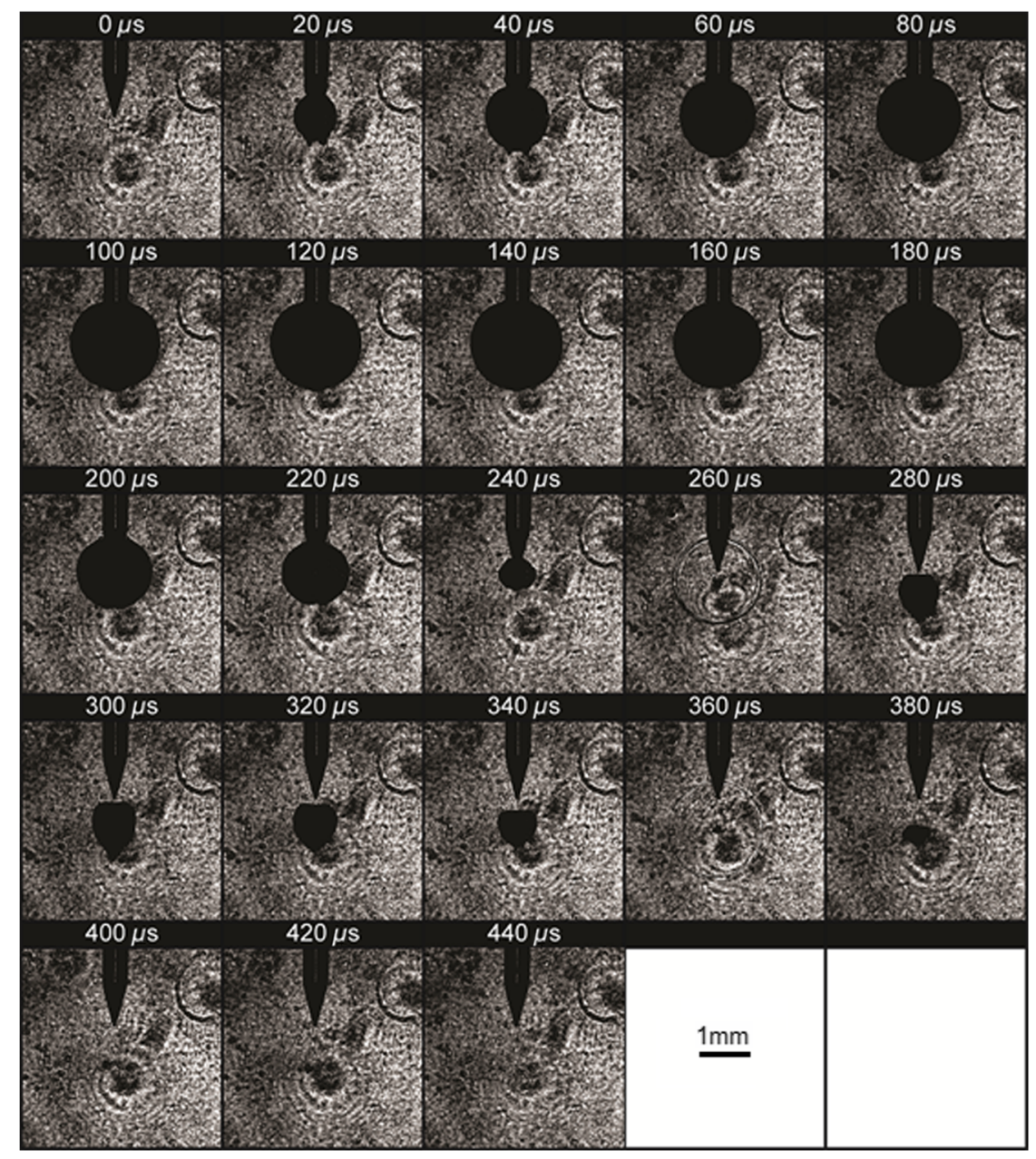

Fig. 2 Typical shadowgraphic images of vapor bubbles in the infinite liquid induced by an Er:YAG laser equipped with a conical FT. 
be obtained as the product of its maximum volume $\left(V_{\max }\right)$ and the hydrostatic pressure $(p)$ of the liquid $\left(p=10^{5} \mathrm{~Pa}\right.$ for water): $:^{2,28}$

$E_{\mathrm{B}}=p V_{\max }$.

\subsection{Measurements of Fluid Motion}

This set of experiments was carried out in a simulated model of a root canal including a narrow lateral canal, designed to represent typical root canal morphology consisting of accessory canals and an apical delta. ${ }^{37-39}$ An accessory canal is defined as a branch of the main pulp canal or chamber that has communication with the external root surface. ${ }^{3}$ Depending on their location, accessory canals can be referred to as furcation canals ${ }^{40}$ when they are in the bifurcation or trifurcation area of the multirooted teeth, ${ }^{41}$ or as lateral canals, when being in the coronal or middle third of the root. ${ }^{3}$ Similarly, an isthmus (anastomosis) is when two or more canals existing in the same root are joined by a thin communication. ${ }^{5,42-44}$

Figure 3 shows the acrylic model of the root canal morphology used in our experiment. The horizontal groove, visible in the bottom-right corner of the model, represents an accessory/lateral canal and was prepared by placing a piece of copper wire on a steel surface, preheated to $80^{\circ} \mathrm{C}$, and pressing the precut model onto it. This method allows for flexibility in the design of the canal and produces very clear, smooth imprints resulting in desirable optical properties. The lateral canal in Fig. 3 is $\sim 13.5 \mathrm{~mm}$ long, with a width of $160 \mu \mathrm{m}$ and a depth of $70 \mu \mathrm{m}$. In all the experiments described, the root canal model was filled with and submerged in distilled water.

The excitation laser sources used in this part of the study were Er:YAG $(\lambda=2.94 \mu \mathrm{m})$ and Nd:YAP $(\lambda=1.34 \mu \mathrm{m})$. The Er:YAG system (LightWalker AT) was equipped with aH14 handpiece with a conical $600 \mu \mathrm{m}$ PIPS FT or a flat ended $600 \mu \mathrm{m}$ VARIAN FT (both manufactured by Fotona). The Nd:YAP system was equipped with an elongated $320 \mu \mathrm{m}$ fiber with a flat ending. The excitation laser pulses were delivered into the root canal model filled with distilled water through a corresponding FT or elongated fiber. The FT's end was placed $h=10 \mathrm{~mm}$ below the water level, as it is schematically shown in the inset of Fig. 3.

The dynamics of the cavitation bubble generated inside the root canal are in many aspects more complex than in the infinite liquid and may depend not only on the depth of the cavity but

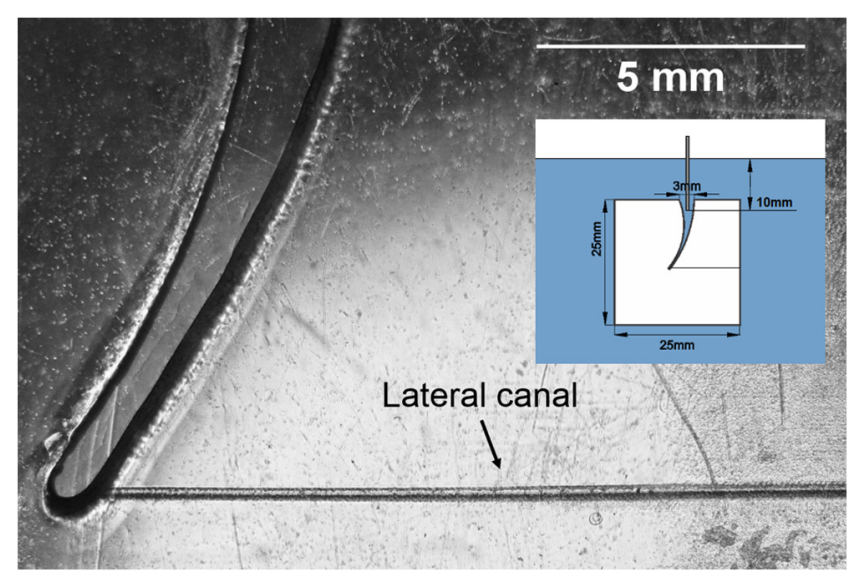

Fig. 3 Acrylic model of a root canal used in the experiments. also on the diameter and shape of the main root canal and its side canals, and as well on the position of the side canals, diameter and shape of the delivery fiber, on the distance of the fiber end from the liquid-air interface, relative depth inside the canal, and other factors. We have studied these dependences extensively with some results already recently published. ${ }^{33}$ The volume of the cavitation bubble decreases with the relative depth of the fiber tip inside the root canal; however, the oscillation times of the bubbles remain roughly the same. Similarly, the dimensions of the cavitation bubbles are affected by the particular shape and size of the root canal, while the oscillation period is largely unaffected by different root canal morphologies. This was tested on cylindrical models of varying radii as well as the different model root canals presented in this paper and Ref. 33. Furthermore, absolute depth (distance, in the range of 5 to $15 \mathrm{~mm}$, of the fiber tip end from the water's surface) has no significant effect on the bubbles' volumes or oscillation periods if the relative depth of the fiber tip inside the root canal model is kept constant. Figure 4 shows a typical cavitation bubble generated with a 25-mJ Er:YAG laser pulse delivered through a conical fiber tip positioned $3 \mathrm{~mm}$ deep inside the model root canal. As it has already been established, ${ }^{33}$ the oscillation period of the bubble is $\sim 200 \%$ to $250 \%$ longer than in an infinite liquid. Due to various factors including friction on the root canal walls, increased volume of water to be displaced from the root canal, and so on, the volume of the vapor bubble inside the model root canal is smaller than the volume of the bubble in an infinite liquid. For reasons mentioned above, the model root canal morphology, the depth of the fiber tip inside the root canal, and the absolute depth of the fiber tip below water surface were all kept constant during the experiments. Further study is required to fully characterize vapor bubbles' dynamics inside different root canal morphologies.

Various approaches were proposed and tested to facilitate the visible detection of water flow. The introduction of ink into the root canal prior to laser agitation proved to serve as a poor indicator of the flow of water, not providing a clear-enough contrast between the colored water entering the lateral canal and the water already present in the lateral canal, and rapidly becoming more diluted with each subsequent laser pulse.

An assortment of small particles ranging from a diameter of under $1 \mu \mathrm{m}$ (polishing powder) to hollow glass spheres with a mean diameter of $10 \mu \mathrm{m}$ used as seeding particles specifically for the purpose of investigating gas flow were also tested and found to be inadequate. While the magnification was not sufficient to make out single particles, they provided good contrast in high-enough concentrations. But their abundance also altered the dynamics of the fluid to such an extent as to be considered incomparable to water. Furthermore, due to the relatively large variability in the size of the glass spheres, a large-enough particle would invariably get pushed into the lateral canal, clogging it and forcing the experimenter to disassemble, clean, and reassemble the model root canal, altering it to such a degree as to make subsequent measurements incomparable to earlier ones.

For monitoring the speed of water flow we utilized, instead of the previously employed methods, gas bubbles that naturally form in a water-filled root canal as a result of laser agitation. While most of the bubbles quickly dissipate, some of them get pushed into the lateral canal and can serve as an indicator of water flow. A high-speed, high-resolution camera (Velociraptor HS by OptoMotive, Mechatronics Ltd., $2048 \times 1088$ pixels, 


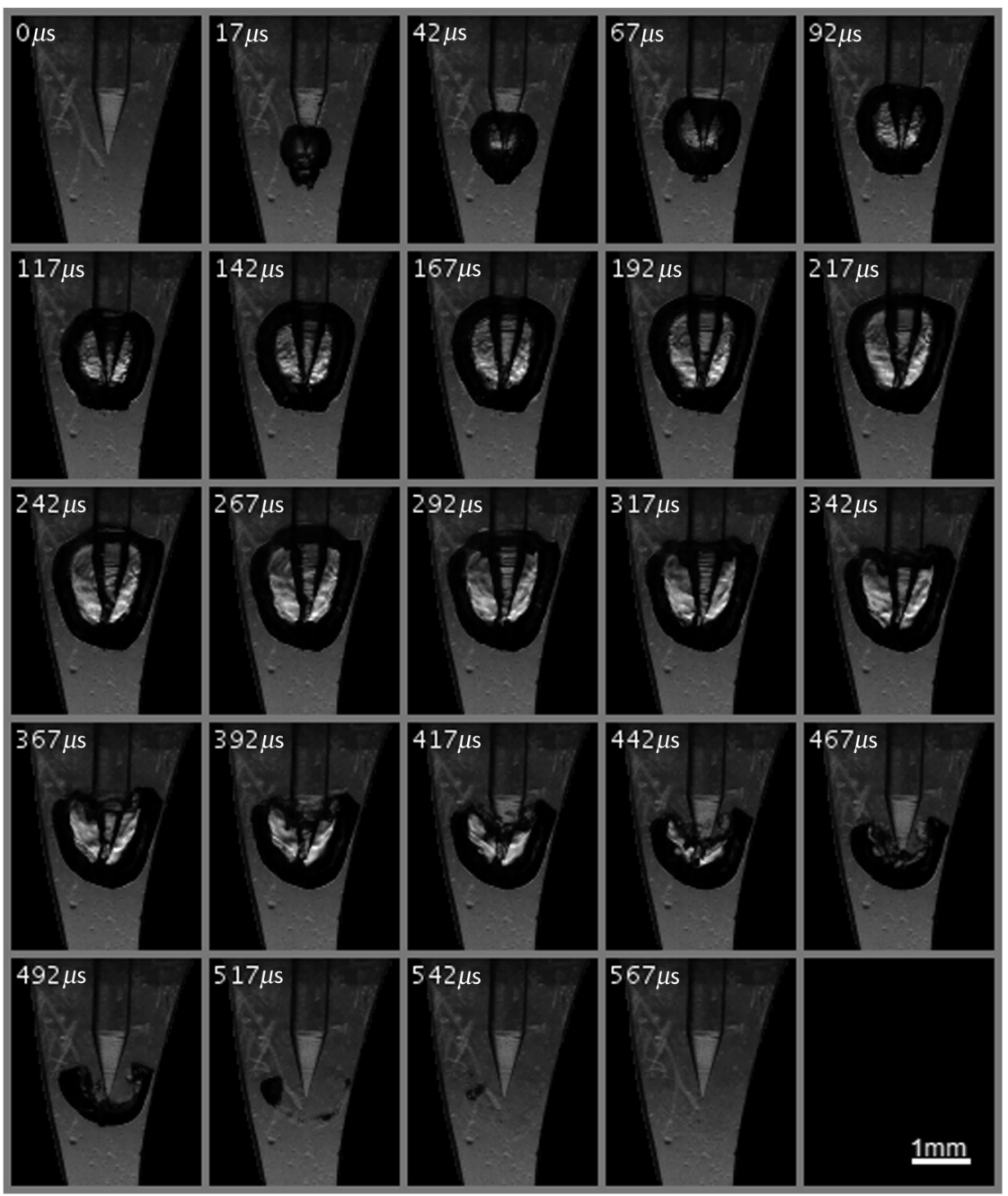

Fig. 4 A sequence of images of a typical cavitation bubble inside the model root canal, generated by an 25-mJ Er:YAG laser pulse delivered through a conical fiber tip. The images were recorded with a highspeed camera (Photron FASTCAM SA-Z) at 120,000 fps. The length of the oscillation is $\sim 550 \mu \mathrm{s}$, which is roughly twice as long as in an infinite liquid (see Fig. 2). The collapse, which initiates at $\sim 280 \mu \mathrm{s}$ after the beginning of the laser pulse, is less symmetrical than is the case in an infinite liquid and rebounds are less pronounced or not present at all.

340 FPS) was used to capture the motion of air bubbles through the lateral canal.

When measuring the dependence of the fluid motion in a lateral canal on Er:YAG laser energy $\left(E_{\mathrm{L}}\right)$, and in order to eliminate any influence of slight changes in the pulse duration with laser system output energy, these measurements were made with the Er:YAG laser system operating at a fixed SSP pulse duration mode and at a fixed system output energy of $130 \mathrm{~mJ}$, resulting in a fixed laser pulse duration of $t_{\mathrm{L}}=85 \mu \mathrm{s}$. The laser energy $\left(E_{\mathrm{L}}\right)$ at the exit of an FT was then varied by inserting apertures of different diameters into the laser handpiece.

On the other hand, when measuring the dependence of the fluid motion on Er:YAG laser pulse duration $\left(t_{\mathrm{L}}\right)$, the system output energy was fixed, and the pulse duration was measured with the InAs photodiode and was varied by changing the pulse duration mode.

Figure 5 shows an example of a pair of air bubbles that are visible in the lateral canal, after each laser pulse. By tracking the position of these bubbles, it was possible to evaluate the dynamics of the flow of water through the lateral canal as a result of PIPS.
By examining light intensities along the length of the lateral canal in individual frames of the recorded video and applying appropriate filters, it is possible to detect and track the position of individual bubbles. Figure 6 shows the intermediate results of the analysis for two typical paths of bubbles at laser frequencies 3 and $4 \mathrm{~Hz}$. The $y$-axis represents the position along the length of the canal (in pixels) and the $x$-axis represents individual frames in the video. The angle of travel depends on the frequency of laser pulses, while the per-pulse speed of the bubble can be determined by examining the movement of the bubbles along the $y$-axis.

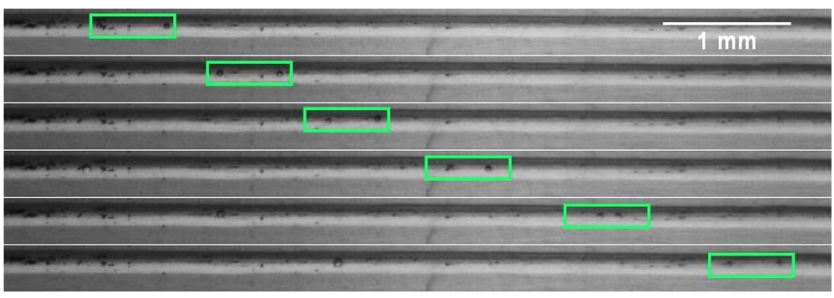

Fig. 5 Movement of a pair of gas bubbles inside the lateral canal. 


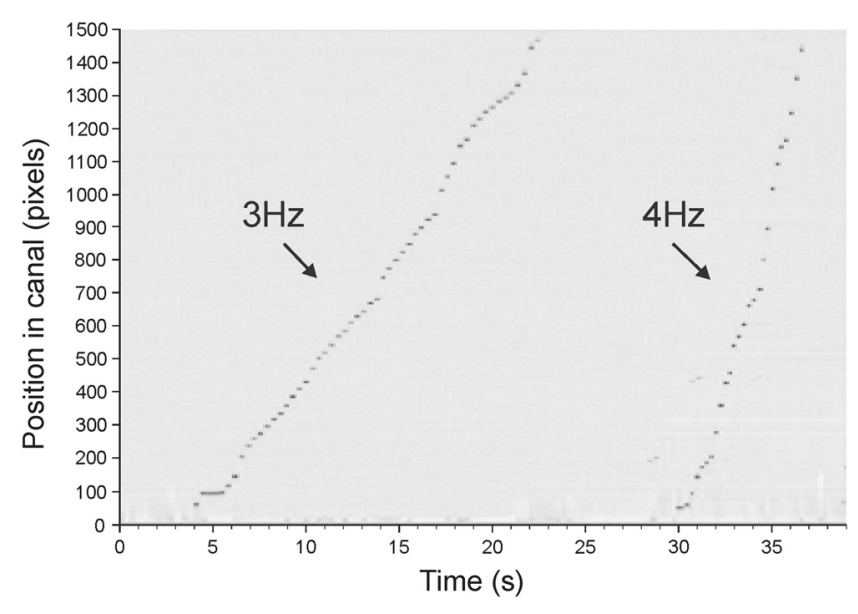

Fig. 6 Bubbles' position versus time at laser frequencies of 3 and $4 \mathrm{~Hz}$.

Figure 7 shows the results of a typical diagram created by applying a peak detection algorithm. The spatial resolution of the system was determined by using images of a calibration pattern. The speed of fluid motion $\left(v_{\mathrm{f}}\right)$, measured in $\mathrm{mm} /$ pulse from the distance traveled by an individual bubble following each laser pulse, was extracted for further analysis.

\section{Results}

\subsection{Analysis of Shadowgraphic Data}

Figure 8 shows the measured maximal radii $\left(R_{\max }\right)$ of cavitation bubbles as obtained for different laser wavelengths, pulse duration modes, and FTs.

The mechanical energies of the bubbles $\left(E_{\mathrm{B}}\right)$ are calculated from bubble's radii (shown in Fig. 8) using Eq. (1) and shown in Fig. 9.

\subsection{Analysis of Fluid Motion Data}

Figure 10 shows the measured dependence of fluid motion per pulse $\left(v_{\mathrm{f}}\right)$ in the lateral canal on Er:YAG $\left(t_{\mathrm{L}}=85 \mu \mathrm{s}\right)$ laser energy for a conical (PIPS) and flat (VARIAN) FTs.

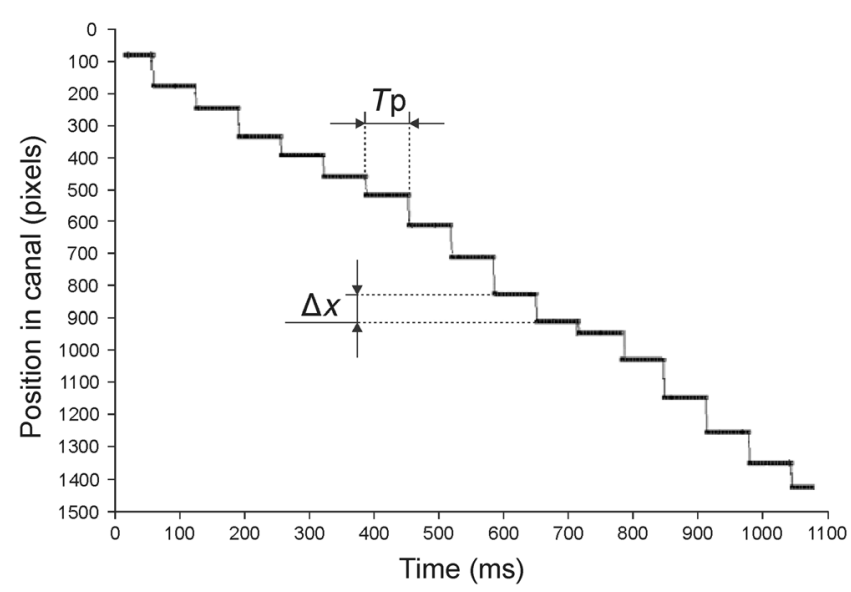

Fig. 7 Peak detection was used to determine bubbles' positions after each laser pulse. $T_{p}$ stands for the period corresponding to the frequency of the laser pulses and $\Delta x$ is the distance traveled by the bubble (in pixels).

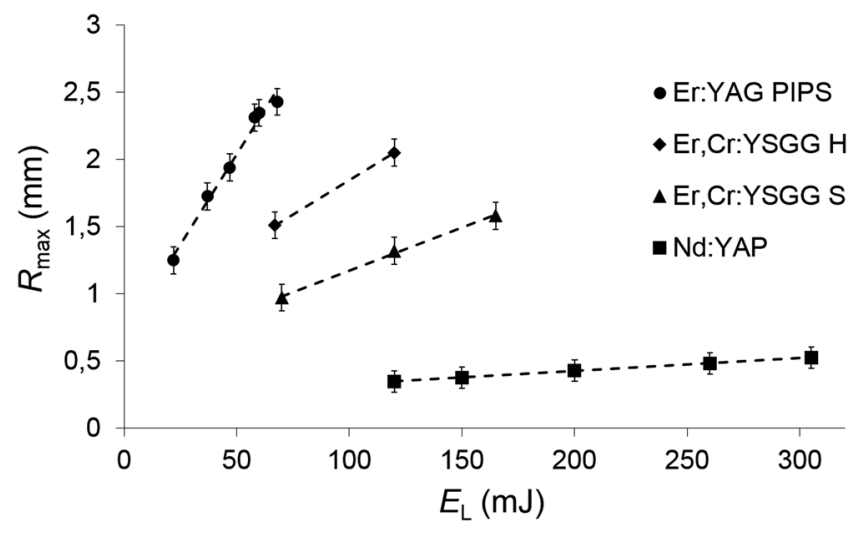

Fig. 8 Measured maximal bubble radii $R_{\max }$ as a function of laser energy $\left(E_{\mathrm{L}}\right)$ for (i) Er:YAG laser pulse with $t_{\mathrm{L}}=80 \mu \mathrm{s}$ (SSP mode) delivered through the $400 \mu \mathrm{m}$ PIPS FT (circles); (ii) Er,Cr:YSGG laser pulse with duration of $t_{\mathrm{L}}=400 \mu \mathrm{s}$ delivered through the 415- $\mu \mathrm{m}$ RFT3 FT (H mode; diamonds) or $t_{\mathrm{L}}=1800 \mu \mathrm{s}$ (S mode; triangles); and (iii) Nd:YAP laser with $t_{\mathrm{L}}=90 \mu \mathrm{s}$ delivered through the $400-\mu \mathrm{m}$ PIPS FT (squares).

The measured dependence of fluid motion $\left(v_{\mathrm{f}}\right)$ on laser pulse duration $\left(t_{\mathrm{L}}\right)$ is shown in Figs. 11 and 12. Figure 11 shows the dependence of $v_{\mathrm{f}}$ on $t_{\mathrm{L}}$ for Er:YAG laser pulses with two different pulse energies: $E_{\mathrm{L}}=30 \mathrm{~mJ}$ and $E_{\mathrm{L}}=40 \mathrm{~mJ}$. Figure 12 shows the measured dependence of $v_{\mathrm{f}}$ on $t_{\mathrm{L}}$ for two types of FTs $(600 \mu \mathrm{m}$ PIPS and $600 \mu \mathrm{m}$ VARIAN) at the same laser pulse energy of $E_{\mathrm{L}}=14.5 \mathrm{~mJ}$.

As can be seen from Figs. 10 and 12, the fluid motion per pulse is larger when a conical FT is used rather than a flat one (see Figs. 10 and 12). The difference is statistically significant $(P<0.0001)$. This is in agreement with a previously published study, ${ }^{28}$ where it was shown that the OD energy-conversion efficiency $\left(\eta_{\mathrm{OD}}\right)$ is higher for a conical FT, and that with a conical FT a spherical bubble develops, while with the flat FT a cylindrically shaped vapor channel gets formed.

Measurements were made also with the Nd:YAP wavelength. Figure 13 shows the measured $v_{\mathrm{f}}$ laser pulse energy for the $\mathrm{Nd}$ : YAP $\left(t_{\mathrm{L}}=90 \mu \mathrm{s}\right)$ laser with a flat, $320-\mu \mathrm{m}$ FT ending.

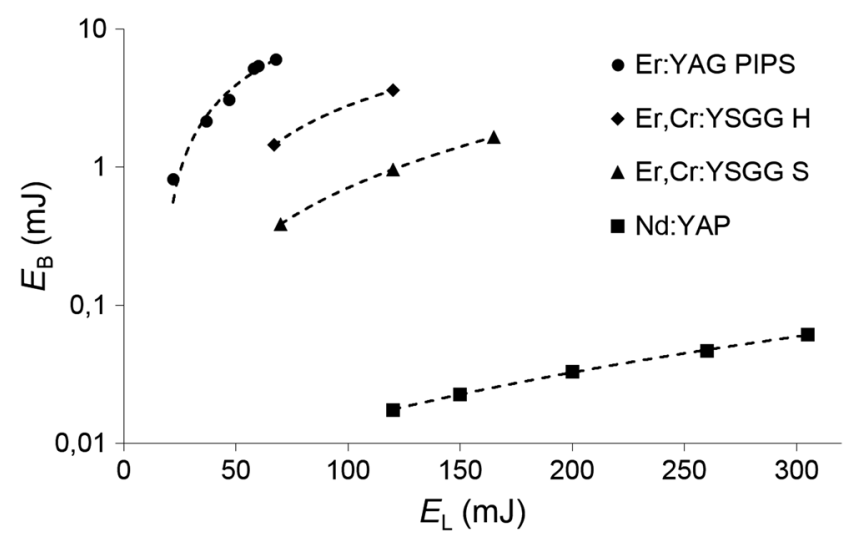

Fig. 9 Bubble energy $E_{\mathrm{B}}$ as a function of laser energy $\left(E_{\mathrm{L}}\right)$ for (i) Er: YAG laser pulse with $t_{\mathrm{L}}=80 \mu \mathrm{s}$ (SSP mode) delivered through the $400-\mu \mathrm{m}$ PIPS FT (circles); (ii) Er,Cr:YSGG laser pulse of duration $t_{\mathrm{L}}=$ $400 \mu$ s delivered through the 415- $\mu \mathrm{m} \mathrm{RFT3} \mathrm{FT} \mathrm{(H} \mathrm{mode;} \mathrm{diamonds)} \mathrm{or}$ $t_{\mathrm{L}}=1800 \mu \mathrm{s}$ (S mode; triangles); and (iii) Nd:YAP laser with $t_{\mathrm{L}}=90 \mu \mathrm{s}$ delivered through the $400-\mu \mathrm{m}$ PIPS FT (squares). 


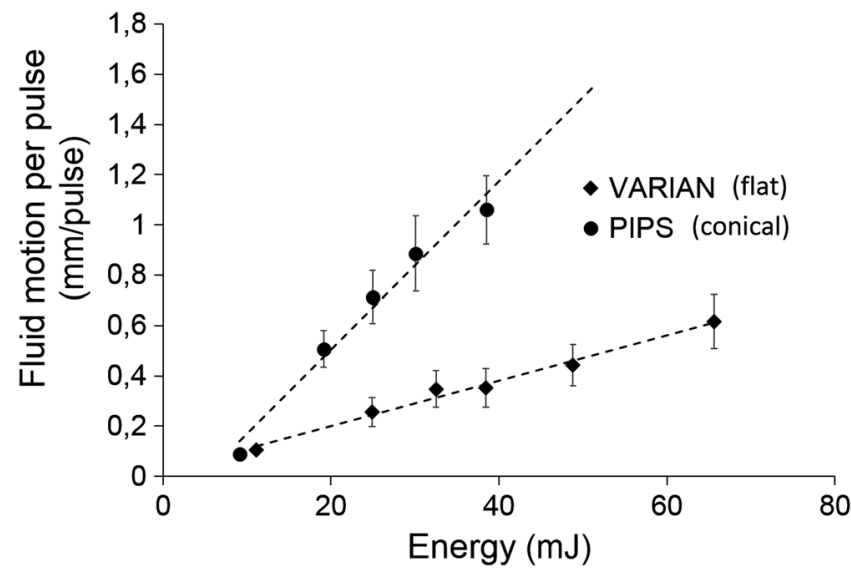

Fig. 10 Measured dependence of fluid motion per pulse $\left(v_{f}\right)$ in the lateral canal on the Er:YAG $\left(t_{\mathrm{L}}=85 \mu \mathrm{s}\right)$ laser pulse energy for a conical (600 $\mu \mathrm{m}$ PIPS) and flat (600 $\mu \mathrm{m}$ VARIAN) FT.

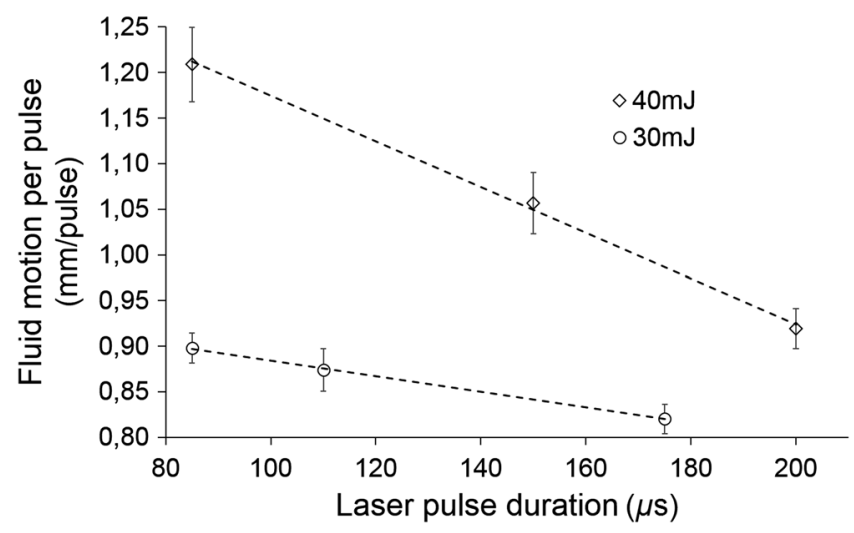

Fig. 11 Dependence of the fluid motion per pulse $\left(v_{f}\right)$ on pulse duration $\left(t_{L}\right)$ for Er:YAG laser pulses with the following two different pulse energies: $E_{\mathrm{L}}=30 \mathrm{~mJ}$ and $40 \mathrm{~mJ}$. A $600-\mu \mathrm{m}$ PIPS FT was used.

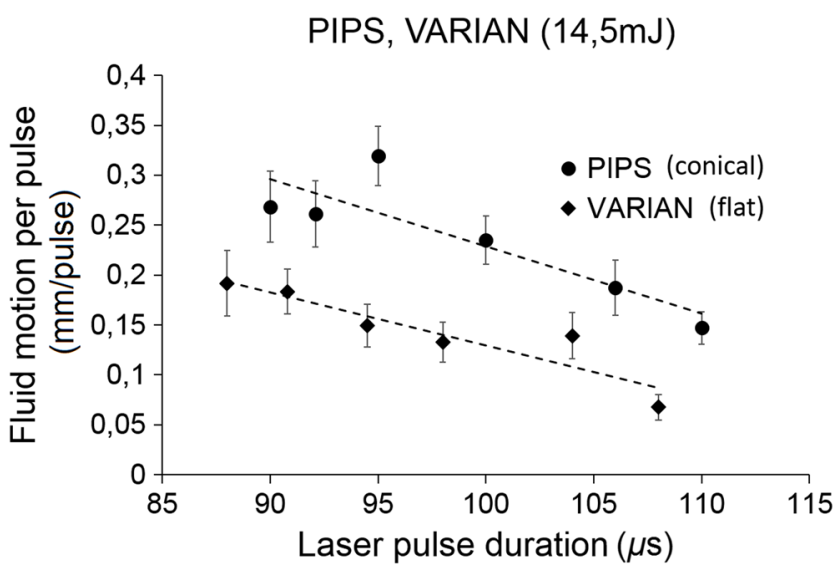

Fig. 12 Measured dependence of the fluid motion per pulse $\left(v_{f}\right)$ on pulse duration $\left(t_{\mathrm{L}}\right)$ for two types of FTs $(600 \mu \mathrm{m}$ PIPS and $600 \mu \mathrm{m}$ VARIAN) at the same laser pulse energy of $E_{\mathrm{L}}=14.5 \mathrm{~mJ}$.

\section{Discussion}

The OD energy-conversion efficiency, defined as $\eta_{\mathrm{OD}}=E_{\mathrm{B}} / E_{\mathrm{L}}$ is reduced by the residual heat that remains deposited in the thin liquid layer extending from the vapor-liquid interface deeper into the liquid. When effects of the thermal diffusion during bubble formation can be neglected, the thickness of this residual

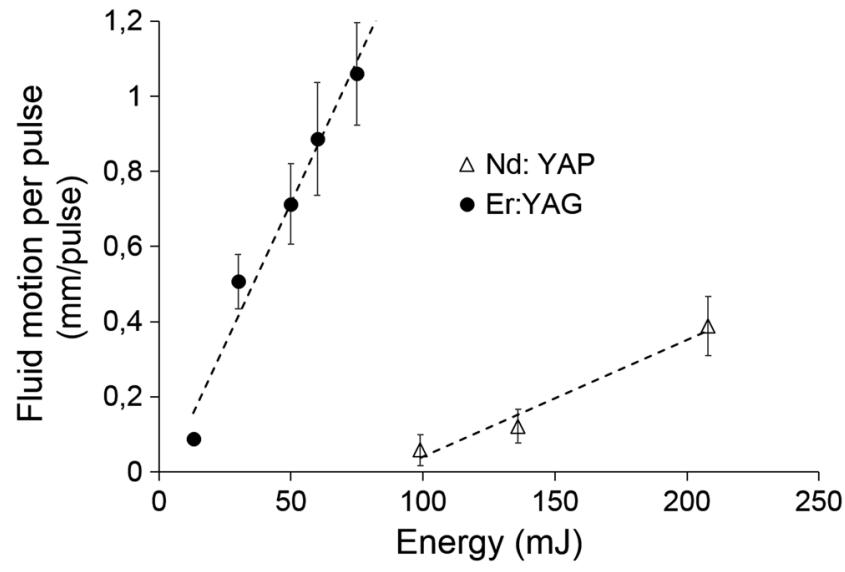

Fig. 13 Measured dependence of the fluid motion per pulse $\left(v_{f}\right)$ on laser pulse energy $\left(E_{\mathrm{L}}\right)$ for the Nd:YAP $\left(t_{\mathrm{L}}=90 \mu \mathrm{s}\right)$ laser with a flat ended $320 \mu \mathrm{m}$ FT. For comparison, the data from Fig. 11 for Er:YAG PIPS are also shown.

heated liquid layer $\left(\delta_{\text {res }}\right)$, and, therefore, the amount of residual heat $\left(H_{\text {res }}\right)$, is expected to be larger for larger optical penetration depth of the laser radiation. Our shadow photography measurements indeed show that the bubble's energy is smaller for wavelengths with larger laser penetration depth $\delta_{\text {opt }}=1 / \mu_{\mathrm{A}}$. The absorption coefficient, $\mu_{\mathrm{A}}$, in water of the tested laser wavelengths ranged from $\mu_{\mathrm{A}}=1200 \mathrm{~mm}^{-1}$ for Er:YAG $(\lambda=2.94 \mu \mathrm{m}),{ }^{45}$ and $\mu_{\mathrm{A}}=400 \mathrm{~mm}^{-1}$ for Er,Cr:YSGG $(\lambda=$ $2.73 \mu \mathrm{m}),{ }^{45}$ to $\mu_{\mathrm{A}}=0.3 \mathrm{~mm}^{-1}$ for Nd:YAP $(\lambda=1.54 \mu \mathrm{m}) .{ }^{46}$ This resulted in $\sim 10$-fold smaller $R_{\max }$, and in $\sim 1000$-fold smaller OD energy-conversion efficiency $\eta_{\mathrm{OD}}=E_{\mathrm{B}} / E_{\mathrm{L}}$ of the Nd:YAP laser in comparison with the Er:YAG laser (see Figs. 8 and 9).

Smaller $\eta_{\mathrm{OD}}$ in comparison to that of the Er:YAG laser was observed also for the Er,Cr:YSGG laser. However, we attribute this difference primarily to the difference in pulse durations and less to the difference in the absorption coefficients. Namely, for longer pulse durations, $\eta_{\mathrm{OD}}$ will be reduced also because of the conductive loss of heat from the laser-water interaction layer, and the residual depth will be increased to $\delta_{\text {res }}=\delta_{\text {opt }}+\delta_{\text {dif }}$. For a spherical heat source, the characteristic diffusion depth $\delta_{\text {dif }}$, to which the liquid temperature is affected during the duration of a laser pulse $t_{\mathrm{L}}$, can be estimated as ${ }^{47}$

$\delta_{\text {dif }}=\left(24 D t_{\mathrm{L}}\right)^{1 / 2}$,

where $D$ stands for the thermal diffusivity of the liquid. Taking into account the thermal diffusivity of water of $D=$ $0.14 \mathrm{~mm}^{2} / \mathrm{s}$, ${ }^{28}$ we obtain for Nd:YAP $\left(t_{\mathrm{L}}=90 \mu \mathrm{s}\right)$, Er:YAG $\left(t_{\mathrm{L}}=80 \mu \mathrm{s}\right)$, Er,Cr:YSGG $\left(t_{\mathrm{L}}=400 \mu \mathrm{s}\right)$, and Er,Cr:YSGG $\left(t_{\mathrm{L}}=1800 \mu \mathrm{s}\right)$ laser pulses the following diffusion depths, respectively: $\delta_{\text {dif }}=17,16,37$, and $82 \mu \mathrm{m}$. For the Nd:YAP laser, the optical absorption length $\left(\delta_{\text {opt }} \approx 3 \mathrm{~mm}\right)$ was much larger than the thermal diffusion length $\left(\delta_{\text {dif }}=17 \mu \mathrm{m}\right)$, and, consequently, the effects of thermal diffusion were negligible. On the other hand, the diffusion depths for the erbium lasers were larger than the penetration depths of $\delta_{\text {opt }}=0.8$ and $2.5 \mu \mathrm{m}$ for Er:YAG and Er,Cr:YSGG, respectively. This indicates that the tested erbium lasers operated in the thermal diffusion regime, ${ }^{47}$ and that their $\eta_{\mathrm{OD}}$ was influenced primarily by the pulse duration and not by the optical absorption coefficient. In the thermal diffusion regime, the residual depth $\left(\delta_{\text {res }}\right)$ can, 


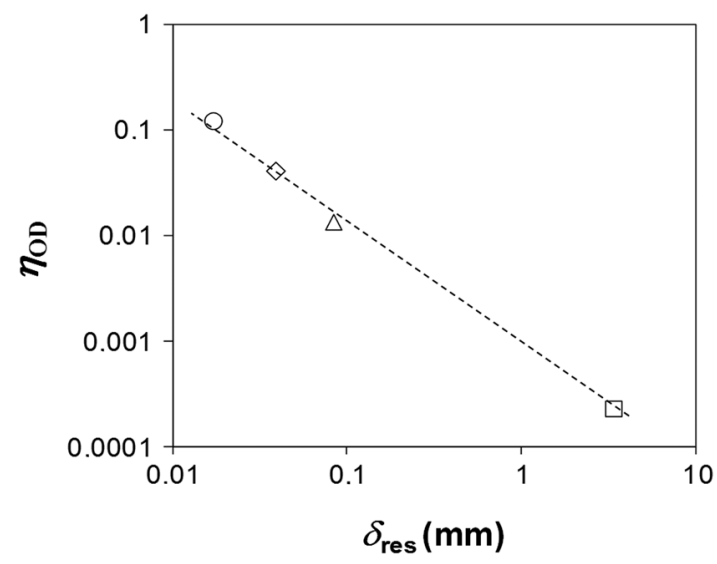

Fig. 14 Measured dependence of the OD energy-conversion efficiency $\left(\eta_{\mathrm{OD}}\right)$ on the residual depth $\delta_{\text {res }}=\delta_{\mathrm{opt}}+\delta_{\text {dif }}$ for Er:YAG with pulse duration $t_{\mathrm{L}}=80 \mu \mathrm{s}$ (circle); Er,Cr:YSGG with $t_{\mathrm{L}}=400 \mu \mathrm{s}$ (diamond); Er,Cr: YSGG having $t_{\mathrm{L}}=1800 \mu \mathrm{s}$ (triangle); and Nd:YAP with pulse duration of $t_{\mathrm{L}}=90 \mu \mathrm{s}$ (square). The dashed line is help to the eye only.

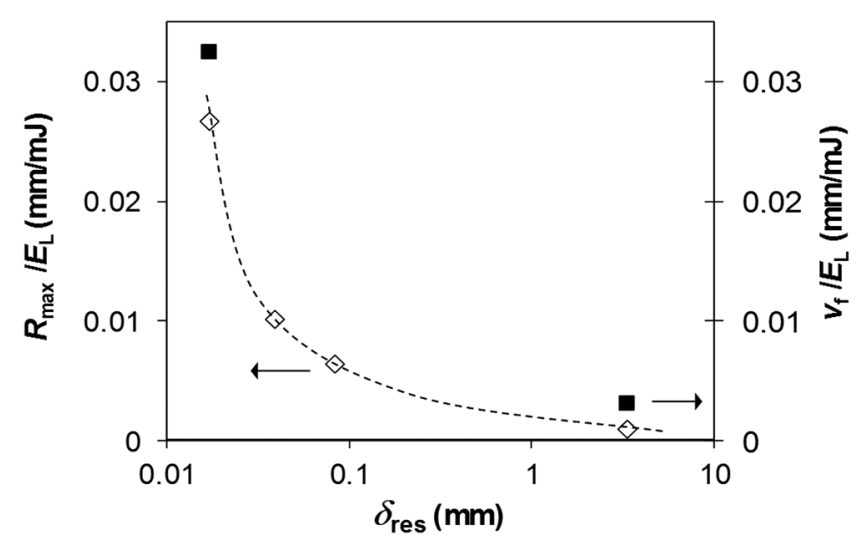

Fig. 15 Measured dependence on the residual depth of the slope efficiencies for maximal radius, $K_{\mathrm{R}}=R_{\max } / E_{\mathrm{L}}$ (open diamonds), and for fluid motion $K_{\mathrm{f}}=v_{\mathrm{f}} / E_{\mathrm{L}}$ (full squares). The dashed curve is help to the eye only.

therefore, be approximated by thermal diffusion lengths $\left(\delta_{\text {dif }}\right)$, while in the optical absorption regime the residual depth can be considered to be represented by $\delta_{\text {opt }}$. The measured dependence of $\eta_{\mathrm{OD}}$ on the residual depth is shown in Fig. 14.

The measured dependence on the residual depth $\left(\delta_{\text {res }}\right)$ of the slope efficiency for maximal radius, $K_{\mathrm{R}}=R_{\max } / E_{\mathrm{L}}$, as obtained from the linear fit to data shown in Fig. 8, and of the slope efficiency for fluid motion $K_{\mathrm{f}}=v_{\mathrm{f}} / E_{\mathrm{L}}$, as obtained from the linear fit to data, shown Fig. 13, is shown in Fig. 15.

As it can be seen from Fig. 15, the dependence on residual depth of the fluid motion within a lateral canal $\left(v_{\mathrm{f}}\right)$ resembles the dependence of the maximal radius as measured in a large water reservoir. Figure 15 thus suggests that the speed of the fluid motion (in $\mathrm{mm} /$ pulse) depends predominantly on the bubble's external dimension and not on the bubble's volume. Our measurements also show that the amplitude of the fluid motion per pulse in a lateral canal is comparable to the maximal bubble's radius in a large water reservoir.

Our fluid motion measurements have also shown that the fluid motion per pulse is smaller for longer pulses. This is in agreement with the observation that the OD energy-conversion efficiency $\left(\eta_{\mathrm{OD}}\right)$ is reduced for longer pulse durations due to larger $\delta_{\text {res }}$ as a result of prolonged heat diffusion (see Fig. 14).
Finally, based on our measurements the cumulative fluid motion achieved during the whole duration $(t)$ of an Er:YAG PIPS procedure seems to be sufficient to achieve a complete irrigation of the lateral canals of the size evaluated in this study, even when very low pulse energies are used. For example, at $E_{\mathrm{L}}=7 \mathrm{~mJ}$ (and therefore $v_{\mathrm{f}}=0.1 \mathrm{~mm} /$ pulse (see Fig. 10), and the repetition rate $f=15 \mathrm{~Hz}$, the cumulative fluid motion, $d_{\text {cum }}=f t v_{\mathrm{f}}$ would in a $t=20 \mathrm{~s},{ }^{22}$ long procedure amount to $d_{\text {cum }}=30 \mathrm{~mm}$, which is longer than any lateral canal, and therefore appears to be sufficient for the irrigation of lateral canals.

During the above procedure performed with an Er:YAG $(\lambda=2.94 \mu \mathrm{m})$ laser, a cumulative (i.e., total) laser energy of $E_{\text {cum }}=2.1 \mathrm{~J}$ is delivered to the root canal, filled with liquid. Assuming that $1 \mathrm{ml}$ of irrigant is placed into the root canal this cumulative energy when converted into heat $\left(H_{\text {res }} \approx E_{\text {cum }}\right)$ would elevate the temperature of the irrigant by $0.5^{\circ} \mathrm{C}$. On the other hand, when using an $\operatorname{Nd:YAP}(\lambda=1.34 \mu \mathrm{m})$ laser source, the pulse energy would have to be increased to $E_{\mathrm{L}} \approx 130 \mathrm{~mJ}$ in order to achieve the same irrigation effect (see Fig. 13). The temperature increase in this case would be $8.0^{\circ} \mathrm{C}$.

\section{Conclusions}

Shadow photography and an air-bubble detection technique were used to study the dependence of the bubble size and speed of fluid motion on laser wavelength, pulse duration, and energy. Assuming that the efficacy of laser-assisted irrigation is correlated with the speed of fluid motion, the obtained experimental data are relevant for optimizing clinical efficacy of laserassisted root canal treatment.

Among the three wavelengths studied (2.94, 2.73, and $1.34 \mu \mathrm{m})$, the Er:YAG $(\lambda=2.94 \mu \mathrm{m})$ laser wavelength was found to be most effective for the formation of cavitation bubbles and to generate the largest fluid motions within narrow lateral canals (the difference is statistically significant at $P<0.0001)$. A comparison between the bubble size and fluid motion data also suggests that when analyzing the influence of different laser parameters on laser irrigation, it is the difference in the bubble's dimension and not in the bubble's volume that leads to the difference in the irrigation efficacy of root canals.

Our study also seems to indicate that using an Er:YAG laser with a short pulse duration and a conical FT, a minimally invasive laser-assisted irrigation can be performed with laser pulse energies below $10 \mathrm{~mJ}$, while still achieving sufficient cumulative fluid motion and irrigation. Further research is required to determine to what extent the findings of this study apply to lateral canals of smaller dimensions than studied in this work.

\section{Acknowledgments}

Authors wish to thank Fotona (www.fotona.com) and Laser and Health Academy (www.laserandhealthacademy.com) for providing laser systems. The authors acknowledge the financial support from the state budget by the Slovenian Research Agency (Programmes No. P2-0392 and P2-0270) and Fotona. Two of the authors (Lukač Nejc and Lukač Matjaž) are currently employees of Fotona.

\section{References}

1. F. Vertucci, A. Seelig, and R. Gillis, "Root canal morphology of the human maxillary second premolar," Oral Surg. Oral Med. Oral Pathol. 38(3), 456-464 (1974). 
2. O. A. Peters, C. I. Peters, and B. Basrani, "Cleaning and shaping the root canal system," Chapter 6 in Cohen's Pathways of the Pulp, K. M. Hargreaves and L. H. Bermann, Eds., 11th ed., pp. 209-279, Elsevier (2016).

3. J. C. Baumgartner, "Endodontic Microbiology," Chapter 16 in Principles and Practice of Endodontics, R. E. Walton and M. Torabinejad, 2nd ed., pp. 277-291 (1996).

4. J. F. Siqueira, Jr. and I. N. Rocas, "Diversity of endodontic microbiota revisited," J. Dent. Res. 88(11), 969-981 (2009).

5. AAE American Association of Endodontists, Glossary of Endodontic Terms, 8th ed., Vol. 45, AAE, Chicago, Illinois (2012).

6. D. McComb and D. C. Smith, "A preliminary scanning electron microscopic study of root canals after endodontic procedures," J. Endod. 1(7), 238-242 (1975).

7. D. R. Fairbourn, G. M. McWalter, and S. Montgomery, "The effect of four preparation techniques on the amount of apically extruded debris," J. Endod. 13(3), 102-108 (1987).

8. C. L. Mader, J. C. Baumgartner, and D. D. Peters, "Scanning electron microscopic investigation of the smeared layer on root canal walls," J. Endod. 10(10), 477-483 (1984).

9. J. E. Barnes, "The production of inlay cavity bevels," Br. Dent. J. 137(10), 379-390 (1974).

10. J. A. Cameron, "The use of ultrasound for the removal of the smear layer. The effect of sodium hypochlorite concentration; SEM study," Aust. Dent. J. 33(3), 193-200 (1988).

11. R. Violich and N. P. Chandler, "The smear layer in endodontics- a review," Int. Endod. J. 43, 2-15 (2010).

12. T. Cengiz, B. O. Aktener, and B. Piskin, "Effect of dentinal tubule orientation on the removal of smear layer by root canal irrigants. A scanning electron microscopic study," Int. Endod. J. 23(3), 163-171 (1990).

13. R. S. Yamada et al., "A scanning electron microscopic comparison of a high volume final flush with several irrigating solutions: Part 3," J. Endod. 9(4), 137-142 (1983).

14. R. R. White, M. Goldman, and P. S. Lin, "The influence of the smeared layer upon dentinal tubule penetration by plastic filling materials," J. Endod. 10(12), 558-562 (1984).

15. J. C. Baumgartner and C. L. Mader, "A scanning electron microscopic evaluation of four root canal irrigation regimens," J. Endod. 13(4), 147-157 (1987).

16. M. Goldman et al., "The efficacy of several endodontic irrigating solutions: a scanning electron microscopic study: Part 2," J. Endod. 8(11), 487-492 (1982).

17. J. C. Yamashita et al., "Scanning electron microscopic study of the cleaning ability of chlorhexidine as a root-canal irrigant," Int. Endod. J. 36(6), 391-394 (2003).

18. J. Blanken et al., "Laser induced explosive vapor and cavitation resulting in effective irrigation of the root canal. Part 1: a visualization study," Lasers Surg. Med. 41(7), 514-519 (2009).

19. R. J. Gentil et al., "Laser induced explosive vapor and cavitation resulting in effective irrigation of the root canal. Part 2: evaluation of the efficacy," Lasers Surg. Med. 41(7), 520-523 (2009).

20. K. Kuhn, "Er:YAG laser activation of sodium hypochlorite for root canal soft tissue dissolution," Lasers Surg. Med. 45(5), 339-344 (2013).

21. O. A. Peters et al., "Disinfection of root canals with photon-initiated photoacoustic streaming," J. Endod. 37(7), 1008-1012 (2011).

22. E. DiVito, O. A. Peters, and G. Olivi, "Effectiveness of the erbium:YAG laser and new design radial and stripped tips in removing the smear layer after root canal instrumentation," Lasers Med. Sci. 27(2), 273-280 (2012).

23. G. Olivi and E. DiVito, "Photoacoustic Endodontics using PIPS"M: experimental background and clinical protocol," J. Laser Health Acad. 2012(1), 22-25 (2012).

24. E. Deleu, M. A. Meire, and R. J. G. De Moor, "Efficacy of laser-based irrigant activation methods in removing debris from simulated root canal irregularities," Lasers Med. Sci. 30(2), 831-835 (2015).

25. G. M. Hale and M. R. Querry, "Optical constants of water in the 200-nm to 200-microm wavelength region," Appl. Opt. 12(3), 555-563 (1973).

26. H. Matsumoto, Y. Yoshimine, and A. Akamine, "Visualization of irrigant flow and cavitation induced by Er:YAG laser within a root canal model," J. Endod. 37(6), 839-843 (2011).
27. A. Vogel and V. Venugopalan, "Mechanisms of pulsed laser ablation of biological tissues," Chem. Rev. 103(2), 577-644 (2003).

28. P. Gregorcic, M. Jezersek, and J. Mozina, "Optodynamic energy-conversion efficiency during an Er:YAG-laser pulse delivery into a liquid through different fiber-tip geometries," J. Biomed. Opt. 17(7), 075006 (2012).

29. P. Gregorcic et al., "Synchronized delivery of Er:YAG laser-pulse energy during oscillations of vapor bubbles, J," J. Laser Health Acad. 2014(1), 14-19 (2014).

30. A. Lloyd et al., "Elimination of intracanal tissue and debris through a novel laser-activated system assessed using high-resolution microcomputed tomography: a pilot study," J. Endod. 40(4), 584-587 (2014).

31. J. D. Koch et al., "Irrigant flow during photon-induced photoacoustic streaming (PIPS) using particle image velocimetry (PIV)," Clin. Oral Invest. 20(2), 381-386 (2016).

32. H. Arslan et al., "Effect of PIPS technique at different power settings on irrigating solution extrusion," Lasers Med. Sci. 30(6), 1641-1645 (2015).

33. N. Lukač, P. Gregorčič, and M. Jezeršek, "Optodynamic phenomena during laser-activated irrigation within root canals," Int. J. Thermophys. 37(7), 1-8 (2016)

34. G. S. Settles, Schlieren and Shadowgraph Techniques, Springer-Verlag, Berlin (2001).

35. P. Gregorčič et al., "In vitro study of the erbium:yttrium aluminum garnet laser cleaning of root canal by the use of shadow photography," J. Biomed. Opt. 21, 015008 (2016).

36. M. Lukač et al., "Influence of water absorption shift on ablation speed of Er:YAG and Er, Cr:YSGG dental lasers," J. Laser Health Acad. 2013(1), 17-22 (2013).

37. J. Guttman, "Prevalence, location and patency of accessory canals in the furcation of molars," J. Periodontol. 49, 21-26 (1987).

38. R. Niemann, G. Dickinson, and R. Jackson, "Dye ingress in molars: furcation to chamber floor," J. Endodont. 19, 293-296 (1993).

39. F. J. Vertucci and R. L. Anthony, "A scanning electron microscopic investigation of accessory foramina in the furcation and pulp chamber floor of molar teeth," Oral Surg. Oral Med. Oral Pathol. 62:319-326 (1986).

40. F. J. Vertucci and R. G. Williams, "Furcation canals in the human mandibular first molar," Oral Surg. Oral Med. Oral Pathol. Oral Radiol. Endod. 38, 308-314 (1974).

41. F. J. Vertucci, "Root canal anatomy of the human permanent teeth," Oral Surg. Oral Med. Oral Pathol. Oral Radiol. Endod. 58, 589-599 (1984).

42. J. V. Cambruzzi and F. J. Marshall, "Molar endodontic surgery," J. Canad. Dent. Assoc. 1, 61-66 (1983).

43. N. R. Weller, S. P. Niemczyk, and S. Kim, "Incidence and position of the canal isthmus. Part 1. Mesiobuccal root of the maxillary first molar," J. Endod. 21, 224-383 (1995).

44. F. J. Vertucci, "Root canal morphology and its relationship to endodontic procedures," Endod. Top. 10, 3-29 (2005).

45. M. Milanic and M. Majaron, "Energy deposition profile in human skin upon irradiation with a 1,342 nm Nd:YAP laser," Lasers Surg. Med. 45, 8-14 (2013).

46. R. R. Anderson and J. A. Parrish, "Selective photothermolysis: precise microsurgery by selective absorption of pulsed radiation," Science $\mathbf{2 2 0}$, 524-527 (1983).

47. B. Majaron et al., "Heat diffusion and debris screening in Er:YAG laser ablation of hard biological tissues," Appl. Phys. B Lasers Opt. 66, 479-487 (1998).

Nejc Lukač is a PhD student at the Faculty of Mechanical Engineering, University of Ljubljana. His main topics of research are the use of lasers and laser pulse shaping in medical applications. Currently, he is an employee of Fotona, d.o.o.

Jure Zadravec: Biography is not available.

Peter Gregorčič received his $\mathrm{PhD}$ in physics from University of Ljubljana, Slovenia, in 2012. Currently, he is working as a scientific collaborator and an assistant professor for laser applications at Faculty of Mechanical Engineering, University of Ljubljana. Within the research fields of optodynamics and laser applications he is 
mainly focused on research and development of laser medicine, laser processing systems and interactions, methods of online measurements, and laser-induced surface microstructures.

Matjaž Lukač holds his MSc and PhD degrees in laser physics. Since 1986 , he has been working as a research associate at Josef Stefan Institute. His main topics of research are the interaction of laser light with biological tissues and physics of laser sources. Currently, he is also managing director of Fotona, d.o.o.

Matija Jezeršek is an associate professor for laser applications at the Faculty of Mechanical Engineering, University of Ljubljana. He is working at the chair of optodynamics and laser applications. He received $\mathrm{a} \mathrm{PhD}$ in mechanical engineering. 